\title{
Cross-Layer Design of Opportunistic Spectrum Access in the Presence of Sensing Error
}

\author{
Qing Zhao*, Lang Tong ${ }^{\dagger}$, Ananthram Swami ${ }^{\ddagger}$, and Yunxia Chen* \\ * Department of Electrical and Computer Engineering \\ University of California, Davis, CA 95616, Email: \{qzhao,yxchen\}@ece.ucdavis.edu \\ ${ }^{\dagger}$ School of Electrical and Computer Engineering \\ Cornell University, Ithaca, NY 15853, Email: 1tong@ece.cornell.edu \\ ¥Army Research Laboratory, Adelphi, MD 20783, Email: aswami@arl.army.mil
}

\begin{abstract}
We address the design of cognitive MAC protocols for opportunistic spectrum access in the presence of sensing error. Decentralized protocols are presented for ad hoc networks where there is no central coordinator or dedicated communication/control channel. Secondary users search for and access spectrum opportunities independently without exchanging local information on spectrum availability. We assume on-demand partial spectrum monitoring subject to sensing errors. Specifically, a secondary user may not be able to simultaneously sense the whole spectrum of interest due to hardware limitation and may only monitor the spectrum when it has data to transmit in order to conserve energy. We propose two approaches to formulating opportunistic spectrum access in the presence of sensing error by considering either a constraint on the probability of colliding with primary users or a penalty imposed on each collision. Under these formulations, we address the joint design of both the spectrum sensor at the physical layer and the cognitive protocol at the MAC layer to maximize spectrum efficiency while limiting the interference to primary users.
\end{abstract}

\section{INTRODUCTION}

Opportunistic spectrum access (OSA), envisioned by the DARPA XG program [1], aims to improve spectrum efficiency by opening licensed spectrum to secondary users to exploit instantaneous spectrum availability. This would allow secondary users to identify and access available spectrum resources provided that the interference to the primary users is kept below a prescribed level.

In this paper, we focus on MAC design for ad hoc OSA networks. Recognizing that there is no central coordinator or dedicated communication/control channel in a secondary ad hoc network, we aim to develop decentralized MAC protocols where each secondary user independently searches for spectrum opportunities without relying on cooperation among secondary users. Furthermore, we do not assume that each secondary user has full knowledge of the availability of all channels; such knowledge implies continuous full-spectrum sensing synchronous among secondary users. While simplifying the design of OSA networks, continuous full-spectrum sensing is energy inefficient and hardware demanding, especially for low-cost battery-powered wireless nodes with bursty traffic. We assume instead that each secondary user can choose to sense a subset of the possible channels (only when it has data to transmit) and must decide if transmission is possible based on the sensing outcome.

Under the assumption of partial spectrum monitoring, we present in this paper a cross-layer approach to OSA that integrates spectrum sensing with spectrum access. We address two fundamental issues in decentralized cognitive MAC under partial spectrum monitoring. The first issue deals with opportunity identification and opportunity exploitation. Our focus here is how spectrum sensing error affects

\footnotetext{
${ }^{0}$ This work was supported by the Army Research Laboratory CTA on Communication and Networks under Grant DAAD19-01-2-0011. Part of this result was submitted to IEEE Journal on Selected Areas in Communications.
}

the overall network performance and how to combat sensing error at both the physical and MAC layers through a joint design of spectrum sensors and OSA protocols. By casting this problem in the framework of Partially Observable Markov Decision Process (POMDP), we obtain spectrum sensing and access strategies as well as the design of spectrum sensor. We point out that although the issue of sensing error has been investigated recently (see [2]), it has not been integrated into the design of cognitive MAC protocols.

The second issue is transmitter-receiver synchronization, which is unique to MAC design in OSA networks. When a secondary user hops in the spectrum, seeking opportunities that are time-varying and location-dependent, its intended receiver needs to hop synchronously in order to carry out the communication. In an ad hoc OSA network with collisions and spectrum sensing errors, maintaining transceiver synchronization without introducing extra control message exchange is nontrivial. We show that the proposed decentralized MAC protocols synchronize opportunistic users in the presence of collisions and sensing errors.

Related Work OSA has received increasing attention [3]. The prevailing approach to OSA tackles network design in two separate steps: (i) opportunity identification assuming continuous full-spectrum sensing; (ii) opportunity allocation among secondary users assuming full knowledge of spectrum opportunities. Opportunity identification in the presence of fading and noise uncertainty has been studied in [2], [4]-[8]. Decentralized opportunity allocation strategies can be found in [9]-[14] and references therein. In [15]-[18], Centralized spectrum sharing protocols with a central coordinator or a dedicated control channel are proposed. Spectrum sharing and power control for a group of interfering users are studied in [19]-[22]. Analytical results on OSA networks can be found in [23] and [24]. In [23], the capacity of multi-hop OSA networks is analyzed using a Markovian model on the spectrum occupancy of the primary users. In [24], the authors identify the impact of channel heterogeneity resulted from OSA on network performance and higher-layer protocol design. This paper is based on results obtained in [25], [26]. The focus of this paper is different formulations of and design strategies for OSA in the presence of spectrum sensing errors.

\section{The Network And Protocol Model}

The Network Model Consider a spectrum consisting of $N$ channels ${ }^{1}$, each with bandwidth $B_{i}(i=1, \cdots, N)$. These $N$ channels are licensed to a primary network whose users communicate according to a synchronous slot structure. The traffic statistics of the primary

${ }^{1}$ Here we use the term channel broadly. A channel can be a frequency band with certain bandwidth. It can also be a collection of spreading codes in a CDMA network or a set of tones in an OFDM system. 
network are such that the occupancy of these $N$ channels follows a discrete-time Markov process with $M=2^{N}$ states $^{2}$. Specifically, the network state in slot $t$ is given by $\left[S_{1}(t), \cdots, S_{N}(t)\right]^{t}$ where $S_{i}(t) \in\{0$ (occupied), 1 (idle) $\}$. We assume that the spectrum usage statistics of the primary network remain unchanged for $T$ slots.

We consider a secondary network seeking spectrum opportunities in these $N$ channels. We focus on an ad hoc network where secondary users join/exit the network and sense/access the spectrum independently without exchanging local information. In each slot, a secondary user chooses a set of channels to sense and a set of channels to access. Limited by its hardware constraints and energy supply, a secondary user can sense no more than $L_{1}\left(L_{1} \leq N\right)$ and access no more than $L_{2}\left(L_{2} \leq L_{1}\right)$ channels in each slot.

Our goal is to develop cognitive MAC protocols for the secondary network. For an ad hoc OSA network without a central coordinator or a dedicated communication/control channel, it is desirable to have a decentralized MAC protocol where each secondary user independently searches for spectrum opportunities, aiming at optimizing its own performance. Such decentralized protocols do not rely on cooperation among secondary users.

The Basic Protocol Structure Without delving into protocol details (which can be found in [25], [27]), we present here the basic protocol structure. At the beginning of each $\operatorname{slot}^{3}$, a secondary user with data to transmit chooses a set of channels to sense and a set of channels to access based on the sensing outcome ${ }^{4}$. Such spectrum sensing and access decisions are made to maximize the spectrum efficiency while limiting the interference to the primary network by fully exploiting the sensing history and the spectrum occupancy statistics. When the secondary user decides to transmit, it generates a random backoff time, and transmits when this timer expires and no other secondary user has already accessed the channel during its backoff time. At the end of the slot, the receiver acknowledges a successful data transmission.

\section{A Decision-Theoretic APPROACH BASED ON POMDP}

In this section, we develop a decision-theoretic approach to MAC design in OSA networks. We show that the OSA network specified in Section II can be modelled by a POMDP and the spectrum sensing and access component of a MAC protocol corresponds to a policy for this POMDP. Existing techniques and results for POMDP can then be used to develop MAC protocols for OSA networks.

\section{A. A POMDP Framework}

Consider the Markovian dynamics of the OSA network specified in Section II. At the beginning of each slot, a secondary user chooses a set $\mathcal{A}_{1}\left(\left|\mathcal{A}_{1}\right| \leq L_{1}\right)$ of channels to sense. Given that the current state of the underlying Markov process is $j$, the user observes $\Theta_{j, \mathcal{A}_{1}} \in$ $\{0,1\}^{\left|\mathcal{A}_{1}\right|}$ which indicates the availability of each sensed channel. Based on this observation, the user chooses a set $\mathcal{A}_{2} \subseteq \mathcal{A}_{1}\left(\left|\mathcal{A}_{2}\right| \leq\right.$ $L_{2}$ ) of channels to access. For the chosen action, the user receives a reward $R_{j, \mathcal{A}_{1}, \mathcal{A}_{2}}$ at the end of this slot. The sequence of operations in each slot is illustrated in Figure 1.

The objective is to choose the sensing and access action $\left\{\mathcal{A}_{1}, \mathcal{A}_{2}\right\}$ sequentially in each slot so that the total expected reward accumulated

\footnotetext{
${ }^{2}$ Since the unavailability of a channel may also be caused by channel fading, the Markov chain model can also include fading statistics.

${ }^{3}$ The slot information can be broadcasted by the primary network.

${ }^{4}$ In a multihop ad hoc networks where realizations of spectrum opportunities are location dependent, spectrum sensing needs to be carried out at both the transmitter and the receiver. Details can be found in [27].
}

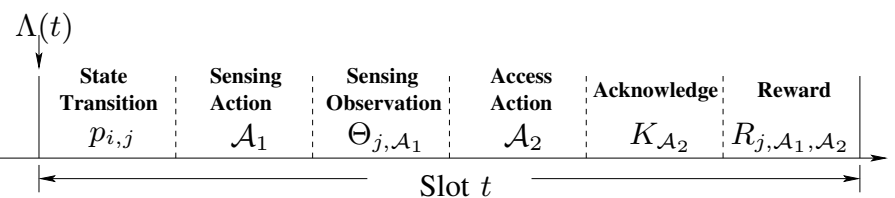

Fig. 1. The sequence of operations in a slot.

over $T$ slots (wherein the spectrum occupancy statistics remain unchanged) is maximized. We now have a POMDP since, in general, the network state cannot be fully observed due to partial spectrum monitoring $\left(\left|\mathcal{A}_{1}\right| \leq L_{1}<N\right)$.

For a POMDP, the internal state of the underlying Markov process is unknown. At the beginning of slot $t$, our knowledge of the internal state of the network based on all past decisions and observations can be summarized by a belief vector $\Lambda(t)=\left[\lambda_{1}(t), \cdots, \lambda_{M}(t)\right]$ where $\lambda_{j}(t)$ is the conditional probability (given the decision and observation history) that the network state is $j$ at the beginning of slot $t$ prior to the state transition (see Figure 1).

It has been shown by Smallwood and Sondik [28] that for any $t$, the belief vector $\Lambda(t)$ is a sufficient statistic for the design of the optimal action $\left\{\mathcal{A}_{1}, \mathcal{A}_{2}\right\}$ in slot $t$. A policy $\pi$ for a POMDP is thus given by a sequence of functions, each mapping from the current belief vector $\Lambda(t)$ to the sensing and access action $\left\{\mathcal{A}_{1}(t), \mathcal{A}_{2}(t)\right\}$ to be taken in slot $t$, i.e.,

$\pi=\left[\mu_{1}, \cdots, \mu_{T}\right], \quad$ where $\mu_{t}: \Lambda(t) \in[0,1]^{M} \rightarrow\left\{\mathcal{A}_{1}(t), \mathcal{A}_{2}(t)\right\}$.

Under this formulation, a spectrum sensing and access strategy is essentially a policy of this POMDP over a finite horizon.

Note that the dimension of the belief vector grows exponentially with the number $N$ of channels. It has been shown in [25] that when channels evolve independently, we can find a sufficient statistic for the optimal policy whose dimension grows linearly with $N$. Specifically, let $\Omega=\left[\omega_{1}, \cdots, \omega_{N}\right]$ where $\omega_{i}$ is the probability (conditioned on the sensing and decision history) that channel $i$ is available at the beginning of a slot. Then $\Omega$ is a sufficient statistic for the optimal OSA protocol under $N$ independent channels.

\section{B. OSA in the Presence of Sensing Error}

In the presence of sensing error, not only the sensing and access strategy but also the operating characteristics of the spectrum sensor affect the performance of the OSA network and the interference perceived by the primary network. The problem is thus a joint design of the spectrum sensor and the OSA MAC protocol.

Spectrum sensors perform a binary hypotheses test: $\mathcal{H}_{0}$ (null hypothesis indicating that the sensed channel is available) vs. $\mathcal{H}_{1}$ (alternative). If the sensor of a secondary user mistakes $\mathcal{H}_{0}$ for $\mathcal{H}_{1}$ (false alarm), the secondary user will refrain from transmitting, and a spectrum opportunity is overlooked. On the other hand, if the detector mistakes $\mathcal{H}_{1}$ for $\mathcal{H}_{0}$ (miss detection), a misidentification of spectrum opportunity occurs; the secondary user collides with a primary user. Let $\epsilon$ and $\delta$ denote, respectively, the overlook (false alarm) and misidentification (miss detection) probabilities. The performance of the sensor is specified by the Receiver Operating Characteristics (ROC) curve which gives $\delta$ as a function of $\epsilon$ (examples are given in Figure 2).

There are two ways of formulating OSA in the presence of sensing error. In the first formulation, the objective is to design the optimal spectrum sensing and access policy $\pi_{*}$ and the operating point $\delta_{*}$ (on the ROC curve) of the spectrum sensor to maximize the 
expected total reward obtained by a secondary user under a constraint on the probability of collision. Specifically, we define the reward $R_{j, \mathcal{A}_{1}, \mathcal{A}_{2}}(t)$ as the number of bits delivered when the secondary user senses channels in $\mathcal{A}_{1}$ and transmits using channels in $\mathcal{A}_{2}$ given that the network is in state $j$. Assume that the number of bits delivered over a channel in one slot is proportional to its bandwidth. The reward is given $b^{5}$

$$
R_{j, \mathcal{A}_{1}, \mathcal{A}_{2}}(t)=\sum_{i \in \mathcal{A}_{2}} S_{i}(t) B_{i}
$$

where $S_{i}(t) \in\{0,1\}$ is the state of channel $i$ in slot $t$.

Let $\zeta$ denote the maximum probability of collision allowed by the primary network. The objective is to design the optimal spectrum sensing and access policy $\pi_{*}$ and the operating point $\delta_{*}$ (on the ROC curve) of the spectrum sensor. Specifically,

$$
\begin{aligned}
\left\{\pi_{*}, \delta_{*}\right\}= & \arg \max _{\{\pi, \delta\}} \mathbb{E}_{\pi}\left[\sum_{t=1}^{T} R_{j(t), \mathcal{A}_{1}(t), \mathcal{A}_{2}(t)} \mid \Lambda(1)\right], \\
& \text { subject to } P_{c} \leq \zeta,
\end{aligned}
$$

where $\mathbb{E}_{\pi}$ represents the conditional expectation given that policy $\pi$ is employed, $P_{c}$ is the probability of collision, and $\Lambda(1)$ the initial belief vector which can be the stationary distribution of the network state. Note that the probability of collision $P_{c}$ depends on the sensing and access policy $\pi$ as well as the operating characteristic of the spectrum sensor.

An alternative formulation is to impose penalty when collisions with primary users occur. Specifically, we define the reward as

$$
R_{j, \mathcal{A}_{1}, \mathcal{A}_{2}}(t)=\sum_{i \in \mathcal{A}_{2}} S_{i}(t) B_{i}-\sum_{i \in \mathcal{A}_{2}}\left(1-S_{i}(t)\right) C_{i},
$$

where $C_{i}>0$ is the cost associated with colliding with a primary user in channel $i$. We thus arrive at an unconstrained formulation where the objective is to maximize the expected total reward accumulated in $T$ slots:

$$
\left\{\pi_{*}, \delta_{*}\right\}=\arg \max _{\{\pi, \delta\}} \mathbb{E}_{\pi}\left[\sum_{t=1}^{T} R_{j(t), \mathcal{A}_{1}(t), \mathcal{A}_{2}(t)} \mid \Lambda(1)\right] .
$$

Note that the penalty $C_{i}$ associated with collision determines the level of interference perceived by the primary network. In this paper, we assume $\left\{C_{i}\right\}_{i=1}^{N}$ is given.

For ease of presentation, we assume in the rest of the paper that $L_{1}=L_{2}=1$. In this case, the action taken in each slot consists of the index $a \in\{1, \cdots, N\}$ of the channel to be monitored and the decision $\Phi_{a} \in\{0$ (no access), 1 (access) $\}$ on whether to transmit. Results obtained in this paper can be readily extended to general cases.

\section{Spectrum Sensing And Access Under the CONSTRAINED FORMULATION}

The problem formulated in (3) is a constrained POMDP which generally requires a randomized optimal policy. To obtain a deterministic strategy with low complexity, we aim at separating the objective function of (3) from the constraint. Specifically, we choose the sensor

\footnotetext{
${ }^{5}$ In this paper, we focus on decentralized cognitive MAC where secondary users make independent and selfish decisions without coordination. In this case, a secondary user chooses its spectrum sensing and access strategies under the assumption that it will receive a reward when the chosen channel is not used by the primary network.
}

operating point based on the constraint on the probability of collision: $\delta_{*}=\zeta$. In this case, the optimal access policy is given by

$$
\Phi_{a}=\left\{\begin{array}{ll}
1 & \text { if } \Theta_{a}=1 \\
0 & \text { if } \Theta_{a}=0
\end{array} .\right.
$$

Since the probability of misidentification of the spectrum sensor is $\zeta$, the probability of colliding with a primary user under this access strategy is $\zeta$, satisfying the design constraint. The problem is then reduced to an unconstrained POMDP where the optimal policy for channel selection is obtained to maximize the throughput of the secondary user.

Both the optimal and suboptimal approaches proposed in [25] under the assumption of perfect sensing can be extended to incorporate overlook and misidentification of spectrum opportunities. We consider here the suboptimal greedy strategy that maximizes the perslot throughput. We assume that channels evolve independently and design the sensing and access strategy based on the reduced-state sufficient statistic $\Omega$.

Assume that channel $i$ transits from state 0 (unavailable) to state 1 (available) with probability $\alpha_{i}$ and stays in state 1 with probability $\beta_{i}$. Let $U_{a}$ denote the number of bits that can be successfully delivered if channel $a$ is chosen in a particular slot. Given that our knowledge of the network state is $\Omega$ at the beginning of this slot prior to the state transition, the channel $a_{*}$ to be selected for maximizing per-slot throughput is given by

$$
\begin{aligned}
a_{*} & =\arg \max _{a=1, \cdots, N} \mathbb{E}\left[U_{a} \mid \Omega\right] \\
& =\arg \max _{a=1, \cdots, N}\left\{B_{a} \operatorname{Pr}\left[S_{a}=1, \Theta_{a}=1 \mid \Omega\right]\right\} \\
& =\arg \max _{a=1, \cdots, N}\left\{B_{a}(1-\epsilon)\left(\omega_{a} \beta_{a}+\left(1-\omega_{a}\right) \alpha_{a}\right)\right\} .
\end{aligned}
$$

At the end of this slot, the belief vector $\Omega$ is updated based on the information gained in this slot. The information gained at the transmitter includes the decision $\left\{a_{*}, \Phi_{a_{*}}\right\}$ and the observation $\left\{\Theta_{a_{*}}, K_{a_{*}}\right\}$ where $K_{a_{*}} \in\{0,1\}$ indicates whether an acknowledgement is received at the end of this $\operatorname{slot}^{6}$. The information gained at the receiver, however, includes only $a_{*}$ and $K_{a_{*}}$ since the receiver does not have the sensing outcome $\Theta_{a_{*}}$ at the transmitter (due to sensing error) and cannot distinguish an unsuccessful transmission from the no-access decision $\Phi_{a_{*}}=0$ of the transmitter. In order for the transmitter and the receiver to arrive at the same belief vector $\Omega(t+1)$, which ensures that they tune to the same channel in the next slot (see (7)), the belief vector should be updated at both the transmitter and the receiver using only $a_{*}$ and $K_{a_{*}}$. The belief vector $\Omega(t+1)$ is thus given by

$$
\begin{aligned}
& \Omega(t+1) \triangleq \mathcal{T}\left(\Omega(t) \mid a_{*}, K_{a_{*}}\right)=\left[\omega_{1}(t+1), \cdots, \omega_{N}(t+1)\right] \\
& \omega_{i}(t+1) \triangleq \operatorname{Pr}\left[S_{i}(t)=1 \mid \Omega(t), a_{*}, K_{a_{*}}\right] \\
& =\left\{\begin{array}{rr}
\omega_{i}(t) \beta_{i}+\left(1-\omega_{i}(t)\right) \alpha_{i}, & \text { if } a_{*} \neq i \\
1, & \text { if } a_{*}=i, K_{a_{*}}=1 \\
\frac{\epsilon\left(\omega_{a_{*}} \beta_{a_{*}}+\left(1-\omega_{a_{*}}\right) \alpha_{a_{*}}\right)}{\epsilon\left(\omega_{a_{*}} \beta_{a_{*}}+\left(1-\omega_{a_{*}}\right) \alpha_{a_{*}}\right)+\left(\omega_{a_{*}}\left(1-\beta_{a_{*}}\right)+\left(1-\omega_{a_{*}}\right)\left(1-\alpha_{a_{*}}\right)\right)} & \text { if } a_{*}=i, K_{a_{*}}=0
\end{array}\right.
\end{aligned}
$$

A detailed derivation of (8) can be found in [26].

We can see from (8) that the transmitter and the receiver maintain the same belief vector independent of collision and sensing error. Since the channel selection is determined by the belief vector, transceiver synchronization is maintained.

\footnotetext{
${ }^{6}$ The transmission of acknowledgement is assumed to be error-free.
} 
The above specifies the spectrum sensing and access strategy when the spectrum sensor operates at $\delta=\zeta$ (see Figure 2). We point out that this approach reduces a constrained POMDP to an unconstrained one by separating the design of the spectrum sensor from that of the sensing and access strategy, resulting in low-complexity but potentially suboptimal design. In our future work, we will pursue the optimal joint design of the spectrum sensor and the OSA MAC and study the tradeoff between complexity and optimality.

\section{Spectrum Sensing And Access Under the UNCONSTRAINED FORMULATION}

In this section, we consider the unconstrained formulation given in (5). The optimization in (5) can be separated into two steps: obtain the optimal policy $\pi_{*}(\delta)$ for a give spectrum sensor $\delta$ and then optimize over $\delta$. We address first the design of the sensing and access strategy for a given sensor. We focus on the greedy approach that aims at maximizing the expected reward in each slot.

We first specify if channel $a$ is chosen, whether the secondary user should transmit based a given sensing outcome. Let $R_{a}$ denote the reward obtained in this slot if the user transmits in channel $a$. Clearly, the optimal access decision $\Phi_{a}$ for sensing outcome $\Theta_{a}=1$ is given by

$$
\mathbb{E}\left[R_{a} \mid \Theta_{a}=1, \Omega\right] \underset{\Phi_{a}=0}{\Phi_{a}=1} 0,
$$

where $\mathbb{E}\left[R_{a} \mid \Theta_{a}=1, \Omega\right]$ can be obtained as

$\mathbb{E}\left[R_{a} \mid \Theta_{a}=1, \Omega\right]$

$=\operatorname{Pr}\left[S_{a}=1 \mid \Theta_{a}=1, \Omega\right] B_{a}-\operatorname{Pr}\left[S_{a}=0 \mid \Theta_{a}=1, \Omega\right] C_{a}$

$=\frac{\left(\omega_{a} \beta_{a}+\left(1-\omega_{a}\right) \alpha_{a}\right)(1-\epsilon) B_{a}-\left(\omega_{a}\left(1-\beta_{a}\right)+\left(1-\omega_{a}\right)\left(1-\alpha_{a}\right)\right) \delta C_{a}\left(\omega_{a} \beta_{a}+\left(1-\omega_{a}\right) \alpha_{a}\right)(1-\epsilon)+\left(\omega_{a}\left(1-\beta_{a}\right)+\left(1-\omega_{a}\right)\left(1-\alpha_{a}\right)\right) \delta}{\left(\omega_{0}\right)}$

The access decision for sensing outcome $\Theta_{a}=0$ can be similarly obtained. The optimal channel selection for maximizing the expected reward in this slot is thus given by

$$
\begin{aligned}
a_{*}= & \arg \max _{a} \mathbb{I}_{\left[\mathbb{E}\left[R_{a} \mid \Theta_{a}=1, \Omega\right]>0\right]} \mathbb{E}\left[R_{a} \mid \Theta_{a}=1, \Omega\right] \operatorname{Pr}\left[\Theta_{a}=1 \mid \Omega\right] \\
& +\mathbb{I}_{\left[\mathbb{E}\left[R_{a} \mid \Theta_{a}=0, \Omega\right]>0\right]} \mathbb{E}\left[R_{a} \mid \Theta_{a}=0, \Omega\right] \operatorname{Pr}\left[\Theta_{a}=0 \mid \Omega\right],
\end{aligned}
$$

where $\left.\mathbb{I}_{[} x\right]$ is the indicator function, $\operatorname{Pr}\left[\Theta_{a}=1 \mid \Omega\right]$ is given by the denominator of (10), and $\operatorname{Pr}\left[\Theta_{a}=0 \mid \Omega\right]$ can be similarly obtained. At the end of this slot, the belief vector $\Omega$ is updated based on $a_{*}$ and $K_{a_{*}}$ as shown in (8).

The above specifies the spectrum sensing and access strategy based on the greedy approach for a given spectrum sensor $\delta$ under the unconstrained formulation. The optimal operating point $\delta_{*}$ can be obtained numerically via Monte Carlo techniques.

\section{NUMERICAL RESUlTS}

In this section, we present numerical results to evaluate the performance of the proposed optimal and suboptimal cognitive MAC protocols. We focus on the constrained formulation.

At the beginning of each slot, the sensor takes $L$ measurements $\left\{Y_{i}\right\}_{i=1}^{L}$ of the chosen channel. We assume that both the channel noise and the signal of primary users can be modelled as white Gaussian processes. We then have

$\begin{cases}\mathcal{H}_{0} \text { (when channel is idle) } & : \quad Y_{i} \sim \mathcal{N}\left(0, \sigma_{0}^{2}\right), i=1, \cdots, L \\ \mathcal{H}_{1} \text { (when channel is busy) } & : \quad Y_{i} \sim \mathcal{N}\left(0, \sigma_{1}^{2}\right), i=1, \cdots, L\end{cases}$

The SNR is given by $\left(\sigma_{1}^{2}-\sigma_{0}^{2}\right) / \sigma_{0}^{2}$. It can be shown that the NeymanPearson detector for this problem is given by the energy detector:

$$
\|\mathbf{Y}\|^{2} \gtrless_{H_{0}}^{H_{1}} \tau \text {. }
$$

The probability $\epsilon$ of false alarm (opportunity overlook) is determined by the threshold $\tau$ :

$$
\epsilon \triangleq \operatorname{Pr}\left\{\|\mathbf{Y}\|^{2}>\tau \mid H_{0}\right\}=1-\Gamma\left(\frac{L}{2}, \frac{\tau}{2 \sigma_{0}^{2}}\right),
$$

where $\Gamma(L, x)=\int_{0}^{x} t^{L-1} e^{-t} d t$ is the incomplete gamma function. The ROC for the Neyman-Pearson testing is thus given by

$$
1-\delta \triangleq \operatorname{Pr}\left\{\|\mathbf{Y}\|^{2}>\tau \mid H_{1}\right\}=1-\Gamma\left(\frac{L}{2}, \eta \frac{\sigma_{0}^{2}}{\sigma_{1}^{2}}\right),
$$

where $\eta$ satisfies $\Gamma\left(\frac{L}{2}, \eta\right)=1-\epsilon$. The ROC curves for different SNRs and numbers $L$ of samples are shown in Figure 2.

In Figure 3 we study the performance of the proposed greedy approach (using the above specified spectrum sensors) as a function of the maximum collision probability $\zeta$ allowed by the primary network. In the upper plot, we focus on the secondary user. We can see that as $\zeta$ increases, the throughput of the secondary user approaches to the performance achieved by the optimal protocol in the absence of sensing errors (the optimal protocol is given in [25]). This is because with a large $\zeta$, the probability $\epsilon$ of overlook can be very small, leading to improved throughput for the secondary user at a price of more collisions with the primary network. As shown in the lower plot, due to frequent collisions, the overall spectrum efficiency considering both the primary and secondary users decreases when $\zeta$ is large. The best spectrum efficiency is obtained when $\zeta$ is around 0.15 .

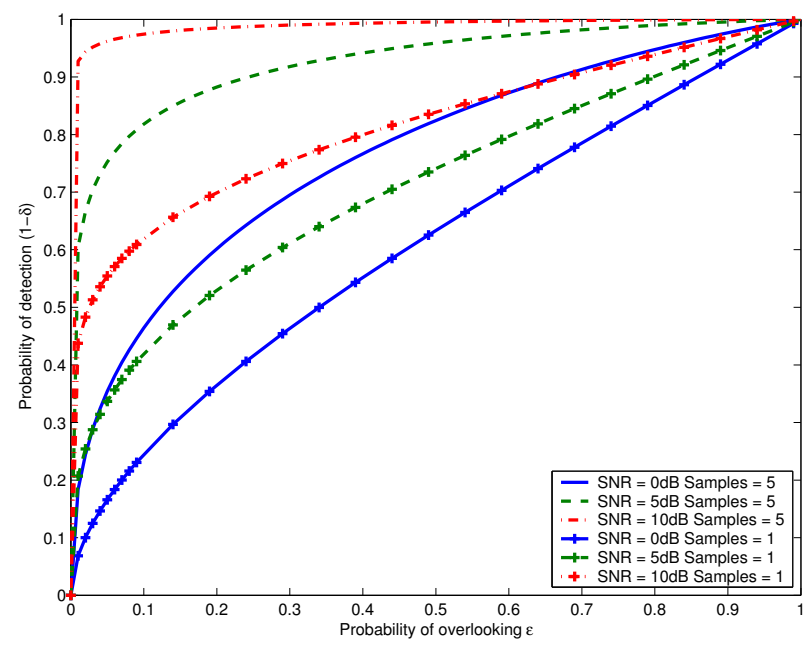

Fig. 2. ROC curves for detecting Gaussian signal in Gaussian noise.

\section{CONCLUSION}

We address in this paper opportunistic spectrum access in the presence of sensing error. Two formulations of this problem are proposed. Under the constrained formulation, the objective is to maximize the throughput of a secondary user subject to a constraint on the maximum probability of collision. For the unconstrained formulation, a penalty is imposed on each collision. Strategies for designing both the spectrum sensor and the OSA MAC protocol are proposed under these two formulations.

\section{REFERENCES}

[1] "DARPA: The Next Generation (XG) Program." http://www.darpa.mil/ato/programs/xg/index.htm. 

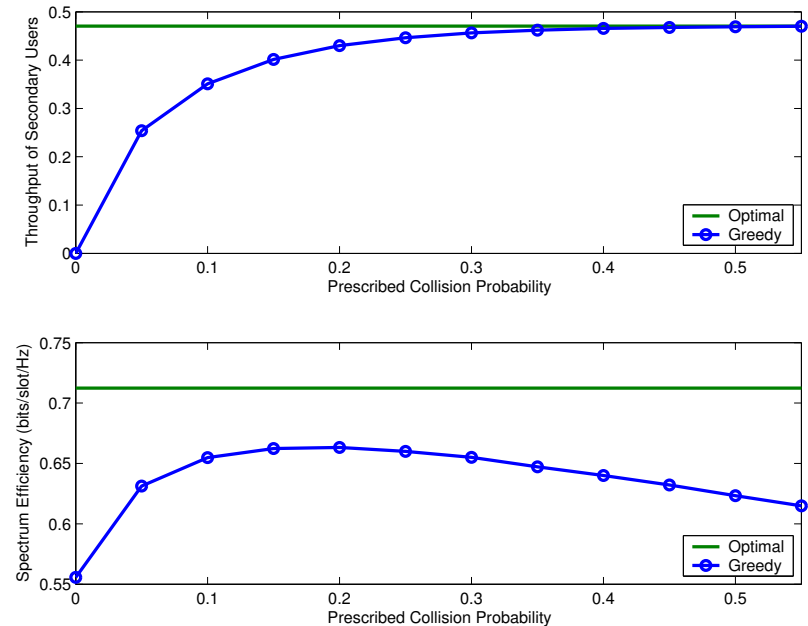

Fig. 3. OSA performance in the presence of sensing error $(N=3$ independent channels with the same bandwidth $B=1$ and transition probabilities $\{\alpha=0.4, \beta=0.5\}$ ).

[2] R. Tandra and A. Sahai, "Fundamental limits on detection in low snr under noise uncertainty," in WirelessCom Symposium on Signal Processing, 2005.

[3] "Proceedings of the first IEEE Symposium on New Frontiers in Dynamic Spectrum Access Networks," November 2005.

[4] K. Challapali, S. Mangold, and Z. Zhong, "Spectrum Agile Radio: Detecting Spectrum Opportunities," in International Symposium on Advanced Radio Technologies, 2004.

[5] G. Ganesan and Y. Li, "Cooperative Spectrum Sensing in Cognitive Radio Networks," in Proceedings of the first IEEE Symposium on New Frontiers in Dynamic Spectrum Access Networks, 2005.

[6] A. Ghasemi and E. Sousa, "Collaborative Spectrum Sensing for Opportunistic Access in Fading Environments," in Proceedings of the first IEEE Symposium on New Frontiers in Dynamic Spectrum Access Networks, 2005.

[7] S. S. N, C. Cordeiro, and K. Challapali, "Spectrum Agile Radios: Utilization and Sensing Architectures," in Proceedings of the first IEEE Symposium on New Frontiers in Dynamic Spectrum Access Networks, 2005.

[8] X. Liu and S. S. N., "Sensing-based opportunistic channel access," $A C M$ MONET (to appear).

[9] S. Mangold, Z. Zhong, K. Challapali, and C. Chou, "Spectrum agile radio: radio resource measurements for opportunistic spectrum usage," in Proc. of Globecom, 2004.

[10] A. Larcher, H. Sun, M. van der Shaar, and Z. Ding, "Decentralized Transmission Strategies for Delay-sensitive Applications over Spectrum Agile Networks ," in Proc. of International Packet Video Workshop, (Irvine, CA), Dec. 2004.

[11] W. Wang, X. Liu, and H. Xiao, "Exploring opportunistic spectrum availability in wireless communication networks," in Proc. of IEEE VTC, 2005.

[12] S. Sankaranarayanan, P. Papadimitratos, A. Mishra, and S. Hershey, "A Bandwidth Sharing Approach to Improve Licensed Spectrum Utilization," in Proceedings of the first IEEE Symposium on New Frontiers in Dynamic Spectrum Access Networks, 2005.

[13] S. Jones, N. Merheb, and I. Wang, "An Experiment for SensingBased Opportunistic Spectrum Access in CSMA/CA Networks," in Proceedings of the first IEEE Symposium on New Frontiers in Dynamic Spectrum Access Networks, 2005.

[14] M. Steenstrup, "Opportunistic Use of Radio-Frequency Spectrum: A Network Perspective," in Proceedings of the first IEEE Symposium on New Frontiers in Dynamic Spectrum Access Networks, 2005.

[15] C. Raman, R. Yates, and N. Mandayam, "Scheduling Variable Rate Links via a Spectrum Server," in Proceedings of the first IEEE Symposium on New Frontiers in Dynamic Spectrum Access Networks, pp. 110-118, 2005.

[16] O. Ileri, D. Samardzija, and N. Mandayam, "Demand Responsive Pricing and Competitive Spectrum Allocation via a Spectrum Server," in
Proceedings of the first IEEE Symposium on New Frontiers in Dynamic Spectrum Access Networks, 2005.

[17] V. Brik, E. Rozner, S. Banerjee, and P. Bahl, "DSAP: A Protocol for Coordinated Spectrum Access," in Proceedings of the first IEEE Symposium on New Frontiers in Dynamic Spectrum Access Networks, 2005.

[18] L. Ma, X. Han, and C. Shen, "Dynamic Open Spectrum Sharing MAC Protocol for Wireless Ad Hoc Networks," in Proceedings of the first IEEE Symposium on New Frontiers in Dynamic Spectrum Access Networks, 2005

[19] J. Huang, R. Berry, and M. Honig, "Spectrum Sharing with Distributed Interference Compensation," in Proceedings of the first IEEE Symposium on New Frontiers in Dynamic Spectrum Access Networks, 2005.

[20] H. Zheng and L. Cao, "Device-centric Spectrum Management," in Proceedings of the first IEEE Symposium on New Frontiers in Dynamic Spectrum Access Networks, 2005.

[21] N. Clemens and C. Rose, "Intelligent Power Allocation Strategies in an Unlicensed Spectrum," in Proc. of the first IEEE Symposium on New Frontiers in Dynamic Spectrum Access Networks, pp. 37-42, November 2005.

[22] R. Etkin, A. Parekh, and D. Tse, "Spectrum Sharing for Unlicensed Bands," in Proceedings of the first IEEE Symposium on New Frontiers in Dynamic Spectrum Access Networks, 2005.

[23] D. Ugarte and A. McDonald, "On the Capacity of Dynamic Spectrum Access Enabled Networks," in Proceedings of the first IEEE Symposium on New Frontiers in Dynamic Spectrum Access Networks, 2005.

[24] P. Kyasanur and N. Vaidya, "Protocol Design Challenges for Multi-hop Dynamic Spectrum Access Networks," in Proceedings of the first IEEE Symposium on New Frontiers in Dynamic Spectrum Access Networks, 2005.

[25] Q. Zhao, L. Tong, and A. Swami, "Decentralized cognitive MAC for dynamic spectrum access," in Proc. of IEEE Symposium on New Frontiers in Dynamic Spectrum Access Networks, Nov. 2005.

[26] Q. Zhao, L. Tong, A. Swami, and Y. Chen, "Decentralized Cognitive MAC for Opportunistic Spectrum Access in Ad Hoc Networks: A POMDP Framework," submitted to IEEE Journal on Selected Areas in Communications, February, 2006.

[27] Q. Zhao, L. Tong, and A. Swami, "A Cross-Layer Approach to Cognitive MAC for Spectrum Agility," in Proc. of Asilomar Conference on Signals, Systems, and Computers, Nov. 2005.

[28] R. Smallwood and E. Sondik, "The optimal control of partially ovservable Markov processes over a finite horizon," Operations Research, pp. 1071-1088, 1971 